\title{
COMMENT
}

Check for updates

\section{Mental health of health-care workers in the COVID-19 era}

Neil Greenberg

Health-care workers involved in the response to the COVID-19 pandemic are often required to work in highly challenging conditions and may therefore be at increased risk of experiencing mental health problems. This Comment sets out a practical approach to protecting the mental health of health-care workers based on contemporary evidence.

These unprecedented circumstances are likely to increase the risk of mental health disorders
The Health Protection Research Unit, King's College London, London, UK. e-mail: Neil.Greenberg@ kcl.ac.uk

https://doi.org/10.1038 s41581-020-0314-5
Throughout the COVID-19 pandemic, many health-care workers (HCWs) have worked extremely long hours in high-pressure environments. Additionally, they may have been exposed to trauma and/or faced moral dilemmas relating to challenges in the delivery of high-quality care, possibly due to a lack of experience or equipment, or as a result of low staffing levels ${ }^{1}$. These unprecedented circumstances are likely to increase the risk of mental health disorders such as post-traumatic stress disorder (PTSD) or depression, other anxiety disorders, substance misuse and suicide. HCWs caring for patients with COVID-19 are also at increased risk of infection, and by extension, have to contend with the risk of infecting their families. Moreover, some individuals, including those of Black, Asian and minority ethnic (BAME) background are at even higher risk of adverse outcomes and may therefore also be at increased risk of psychological issues. The risk of psychological harm is not restricted to frontline HCWs; in fact most HCWs, regardless of their speciality, are likely to have experienced notable challenges since the pandemic began, including changes to the way they work and, at times, being unable to deliver the quality of care they would normally. Whilst some HCWs will undoubtedly thrive in such stressful circumstances, it is imperative that managers of HCWs take measures to protect the mental health of staff as well as identify those who do experience psychological injuries, to ensure they are provided with appropriate evidence-based support or care.

The vast scientific literature on the psychological consequences of exposure to trauma shows that the two risk factors most strongly predictive of long-term mental health status are lack of post-trauma social support and exposure to stressors during recovery from trauma ${ }^{2}$. These stressors might be directly attributable to the trauma, for example, the death of a family member or co-worker, or secondary to the crisis, such as relationship difficulties ${ }^{3}$. The good news is that health-care managers can very strongly influence a HCW's experience of being supported and their exposure to workplace pressures during the post-crisis period. Studies of HCWs dealing with previous infectious disease outbreaks show the powerful effect that supportive managers have on the mental health of their staff ${ }^{4}$, whereas evidence from military studies show that a phased return to 'normal' work is also beneficial.

Taken as a whole, available evidence suggests that protecting the mental health of HCWs requires consideration of six key elements. First, HCWs should be appropriately thanked. Appropriate acknowledgement of the challenging work undertaken can foster resilience 5 . This acknowledgement should also include recognition of potential psychological difficulties and provide information about support options available.

Second, given that avoidance is a key symptom of traumatic stress and this behaviour may manifest through distressed staff staying away from work, it is imperative that staff who do not turn up to work are contacted in case their non-attendance is indicative of poor mental health.

Third, as the COVID-19 pandemic begins to recede all HCWs should receive 'return to normal work' interviews. These should happen as staff begin the transition from crisis response roles back to the new normal and should be conducted by supervisors who, by virtue of experience or training, can confidently speak about mental health. They provide an opportunity for a supervisor to better understand a staff member's experiences and can help foster an 'it's okay not to be okay' approach to mental health. Such discussions have been shown to reduce absences due to sickness in other trauma-exposed occupations $s^{6}$.

Fourth, managers should pay particular attention to HCWs in high-risk groups, such as those with a BAME background, and junior or inexperienced staff who have been working above their expected level of competence. The abovementioned 'return to normal work' interview is again important here, as it can be used to identify the presence of pertinent stressors such as bereavement or feelings of being overwhelmed. Particular attention should be paid to staff who, during the crisis, have worked far beyond their usual role, for instance, 


It is
imperative that
managers of
[health-care
workers] take
measures to
protect the
mental health
of staff

an outpatient nurse who may have been required to care for a ventilated patient during the crisis. Strong evidence shows that additional stressors can have a very important impact on the mental health of people who have already suffered a traumatic experience?

Fifth, as recommended by the UK National Institute for Health and Care Excellence, anyone who has been exposed to a potentially traumatic event should be actively monitored, particularly those considered at higher risk of developing mental health problems. Although this type of monitoring forms part of good management, available evidence suggests that proactively asking such individuals about their mental health can increase the take-up of mental health care as has been found after terrorist incidents in the $\mathrm{UK}^{2}$. This monitoring could be achieved through the use of an anonymous, online self-check tool comprising a range of mental health measures giving tailored advice, such as self-help information or ways to access professional care. Such an anonymous approach is likely to increase the chance that HCWs will use the self-check tool.

Lastly, given the likelihood that HCWs have been exposed to morally distressing circumstances during the COVID-19 pandemic, most probably repeatedly, managers should help them to make sense of their experiences. Being able to develop meaningful narrative that does not blame themselves or others for what happened during the crisis is likely to reduce the risk of them suffering psychological harm. One evidence-based model that may prove useful is Schwartz rounds - a structured forum in which staff can discuss emotional and social aspects of work which has been shown to help staff communicate better in both US and UK health-care settings.

Supporting the mental health of HCWs is not just morally justified - done well it should lower the risk of mental illness and maximize the opportunity for staff to experience psychological growth ${ }^{8}$ from overcoming the challenges faced during the pandemic. As described previously, the unwritten psychological contract between HCWs, their managers and the public has been that staff members will give their all to save lives and in return the nation does all it can to protect their mental health through the provision of proper support ${ }^{9}$. Although rapid access to effective evidence-based treatments will be needed for an important minority of HCWs as a result of the stressors experienced during the COVID-19 pandemic, a substantial evidence base supports the notion that health-care managers have a substantial role in protecting the mental health of their staff as well as in helping those that need additional care and support to access it.

1. Greenberg, N. et al. Managing mental health challenges faced by healthcare workers during covid-19 pandemic. Br. Med. J. 368 $\mathrm{m} 1211$ (2020).

2. Brewin, C. R. et al. Outreach and screening following the 2005 London bombings: usage and outcomes. Psychol. Med. 40, 2049-2057 (2010).

3. Tempest, E. L. et al. Secondary stressors after flooding: a cross-sectional analysis. Eur. J. Public Health 27, 1042-1047 (2017).

4. Brooks, S. K. et al. A systematic, thematic review of social and occupational factors associated with psychological outcomes in healthcare employees during an infectious disease outbreak. J. Occ. Environ. Med. 60, 248-257 (2018).

5. McCanlies, E. C. et al. The effect of social support, gratitude, resilience and satisfaction with life on depressive symptoms among police officers following Hurricane Katrina. Int. J. Soc. Psychiatry 64, 63-72 (2018)

6. Milligan-Saville, J. S. et al. Workplace mental health training for managers and its effect on sick leave in employees: a cluster randomised controlled trial. Lancet Psychiatry 4, 850-858 (2017).

7. Lock, S. et al. Secondary stressors and extreme events and disasters: a systematic review of primary research from 2010-2011. PLoS Curr. https://doi.org/10.1371/currents.dis. a9b76fed 1 b2dd5c5bfcfc 13c87a2f24f (2012).

8. Brooks, S. K. et al. Psychological resilience and post-traumatic growth in disaster-exposed organisations: overview of the literature. BMJ Mil. Health 166, 52-56 (2020).

9. Greenberg, N. "Going for Growth". An outline NHS staff recovery plan post-COVID19 (outbreak 1). Royal College of Psychiatrists. https://www.rcpsych.ac.uk/docs/default-source/about-us/covid-19/ going-for-growth-version-3-05-05-20.pdf?sfvrsn=7cf71 c97_4 (2020).

\section{Acknowledgements}

The author's work is funded by the National Institute for Health Research Health Protection Research Unit (NIHR HPRU) in Emergency Preparedness and Response, a partnership between Public Health England, King's College London and the University of East Anglia. The views expressed are those of the author and not necessarily those of the NIHR, Public Health England or the Department of Health and Social Care.

\section{Competing interests}

N.G. runs a psychological health consultancy called March on Stress Ltd. 